\title{
Expandable liver organoids generated from human chemically derived hepatic progenitor enable alcoholic liver modeling
}

\author{
Yohan Kim, Sangtae Yoon, Myoung-Hoi Kim, Da Hee Hong, Taehun Kim, Jaemin Jeong, Dongho Choi
}

Division of Hepatobiliary, Department of Surgery, Hanyang University College of Medicine, Seoul, Korea

Background: Liver organoids, an attracting source for studying cell to cell interaction, drug screening, and disease modeling in three-dimension systems were demonstrated to be developed from $\mathrm{EpCAM}^{+}$cells. Our group previously reported chemically derived hepatic progenitors $(\mathrm{hCdHs})$, reprogrammed from human primary hepatocytes with bi-potent differentiation capacity and $\mathrm{EpCAM}^{+}$feature. Therefore, we aimed to generate organoids showing characteristics of liver organoids using hCdHs.

Methods: To generate hCdHs, human primary hepatocytes were cultured with reprogramming medium including HGF, A83-01 and CHIR99021 for 7 days. Isolated human liver cells and hCdHs were cultured on Matrigel with organoid medium to generate human adult liver organoids and hCdHs derived liver organoids ( $\mathrm{hCdHO}$ ).

Results: hCdHO were morphologically undistinguished and showed high generation efficiency compared with human adult liver organoids. hCdHO were stably expanded over a 6-month period and expressed liver organoid-specific markers. hCdHO cultured in hepatic differentiation medium showed increased expression of hepatic markers and functional capacity such as CYP activity. Upon transplantation in FRG mice models, hCdHO effectively repopulated the injured liver. We further developed an alcoholic liver model using hCdHO cultured in hepatic differentiation medium under ethanol treatment which presented us with alcoholic liver disease associated alternations in mitochondrial membrane potential.

Conclusions: Our studies suggest that hCdHO have a potential to be a novel liver organoid cell source for performing disease modeling.

Corresponding author: Dongho Choi

E-mail: crane87@hanyang.ac.kr

\section{(c) The Korean Society for Transplantation}

This is an Open Access article distributed under the terms of the Creative Commons Attribution Non-Commercial License (http://creativecommons.org/licenses/by-nc/4.0/) which permits unrestricted non-commercial use, distribution, and reproduction in any medium, provided the original work is properly cited. 